\title{
Protecting the infant-parent relationship: special emphasis on perinatal mood and anxiety disorder screening and treatment in neonatal intensive care unit parents
}

\author{
Julie Johnson Rolfes (D) $^{1 凶}$ and Megan Paulsen (iD) ${ }^{1}$ \\ (c) The Author(s), under exclusive licence to Springer Nature America, Inc. 2021
}

Perinatal mood and anxiety disorders (PMADs) are common, particularly among parents of infants requiring admission to the neonatal intensive care unit (NICU), yet remain underdiagnosed and undertreated. Undertreated parental mental health disorders can interfere with healthy infant development, compounding abnormal neurodevelopment and psychosocial development that preterm or ill newborns may already face. Interdisciplinary efforts to increase PMAD awareness, screening, and referral uptake may improve family-infant health and developmental outcomes in high-risk infants requiring NICU admission. Therefore, special emphasis on PMAD screening and treatment in NICU parents aligns with the American Academy of Pediatrics mission and should be a focus in neonatal care and included in education, quality improvement, and outcome-based research initiatives.

Journal of Perinatology (2022) 42:815-818; https://doi.org/10.1038/s41372-021-01256-7

\section{INTRODUCTION}

We are neonatologists, mothers whose own infants have required neonatal intensive care, and have been personally impacted by perinatal mood and anxiety disorders (PMADs) [1]. We know, first hand, the benefits of PMAD awareness, screening, and access to treatment, as well as several shortfalls in our current neonatal intensive care unit (NICU) systems related to this important topic. While we see a growing body of literature around PMADs, with increasing attention toward mental health initiatives, we also see families every day who have fallen through the cracks of our care system, and well-intentioned colleagues unaware of the gravity of the problem and/or how to help. So, we want to help spark momentum around PMAD awareness and intervention specifically within the neonatology community. We want to accelerate the progress that we, as a nation of neonatal care providers, can make in supporting our NICU families through changes in NICU care models and additional research into the impact of PMADs on our developing infants.

\section{EPIDEMIOLOGY}

Perinatal mood and anxiety disorders, which develop during pregnancy or within a year following birth, affect at least $15-20 \%$ of pregnant and postpartum women [2]. Rates of PMADs are particularly high among parents of infants admitted to the NICU. Compared to parents of well newborns, NICU parents have a $20-30 \%$ higher prevalence of PMADs [3-12]. Unfortunately, epidemiologic studies estimate that $50-70 \%$ of PMADs go undiagnosed, and for the minority who are able to receive an appropriate PMAD diagnosis, only $50 \%$ are able to access adequate treatment [13].
Untreated or undertreated PMADs are associated with worse physical health outcomes for both mothers and their children, including severe maternal morbidity and mortality in the first year postpartum [2, 13-17]. Postpartum depression represents the greatest risk factor for maternal suicide and infanticide [13]. Neonatologists should be aware that children of mothers with PMADs have increased risk of prematurity, sudden infant death, and need for neurodevelopmental and neurobehavioral support services $[16,18]$. A study based on a 2017 birth cohort in the United States projected that PMADs would cost \$14 billion dollars from conception to 5 years postpartum [16]. The average estimated cost per affected mother-child dyad was $\$ 31,800$, more than one third of which was incurred by the children's care costs [16].

In acknowledgement of the scope and gravity of PMADs, the American Academy of Pediatrics (AAP), the American College of Obstetricians and Gynecologists, and the U.S. Preventive Services Task Force uniformly recommend screening of postpartum women for depression as a component of quality maternal-child healthcare $[2,17,19]$. Despite this, we have often encountered families in the NICU who are suffering from undiagnosed and/or untreated PMADs. We would like to share some of the observations that we have made from both our personal and professional experience with PMADs, and how we see care improving for NICU families with PMADs.

\section{ENHANCED EDUCATION TO IMPROVE PMAD AWARENESS}

We believe that the low level of diagnosis and treatment for PMADs in the NICU partly stems from a lack of recognition of the problem by families and care providers alike, as well as the stigma of mental illness diagnoses, often exacerbated by gender identity, structural vulnerabilities, and cultural perceptions of PMADs

${ }^{1}$ Department of Pediatrics, Division of Neonatology, University of Minnesota Medical School, Minneapolis, MN, USA. ${ }^{\circledR e m a i l: ~ r o l f e 030 @ u m n . e d u ~}$ 
$[20,21]$. Education to improve mental health literacy can lower these barriers [20-23]. Hall et al. have described their experience of improving their NICU staff's knowledge of and attitude towards psychosocial support of NICU parents following the completion of a one-hour online module focused on NICU parent PMADs $[22,23]$. It is reassuring that just an hour of education can make a measurable impact on NICU care providers, but we acknowledge that the work of culture shift will take on-going efforts and broad engagement.

We believe that an understanding of the long-lasting effects of psychological trauma on the human body for generations may allow some NICU care providers and families to better appreciate the importance of identifying risk factors for PMADs, and provide support for all families in the NICU through trauma-informed care $[24,25]$. By integrating trauma-awareness into the care model of the NICU, we can normalize the physical response to stress and the risk of its toxicity for our NICU patients, families and caregivers. This could help propel the destigmatization of PMADs.

We applaud efforts from the American Board of Pediatrics and the Accreditation Council on Graduate Medical Education to build mental health training into the curriculum of pediatric residents and neonatal-perinatal medicine (NPM) fellows [26, 27]. These newly trained physicians can help become champions for PMAD educational initiatives, as a group of NPM fellows on the AAP Section on Neonatal Perinatal Medicine, Training and Early Career Neonatologist Advocacy Committee have with a recent NICU Mental Health Campaign.

To further the national attention on this issue and more rapidly spread acceptance of the need for comprehensive PMAD identification and treatment, we recommend the AAP Committee on the Fetus and Newborn and/or AAP Committee on Psychosocial Aspects of Child and Family Health release updated policy reports, spotlighting how a mental health workforce based in the NICU could make a significant, positive public health impact for NICU patients and their families.

Ultimately, to maximize the rate and breadth of a culture shift toward PMAD acceptance and effective support, we will need to employ a combination of educational opportunities targeting various stakeholders including physicians, advanced practice providers, nurses, developmental therapists, lactation specialists, social workers and spiritual health providers, as well as NICU families.

\section{IMPROVING NICU FAMILY SCREENING TO INCREASE PMAD DIAGNOSIS}

We believe that significant gaps in the structure and consistency of PMAD screening within the NICU also contribute to underdiagnosis. To improve, we first need to widen the scope of symptoms that are screened for. The umbrella term PMAD includes postpartum depression (PPD), but also anxiety, post-traumatic stress disorder (PTSD), obsessive compulsive disorder, and others [14, 28]. While postpartum depression and anxiety remain the most common types of PMAD in NICU families, other types of PMAD are not infrequent. NICU parents have a higher rate of PTSD, in particular, as compared to parents of well newborns [3-6, 8, 10,29]. As a result, a broader range of symptom surveillance will be needed to effectively identify those with different types of PMAD.

We next suggest expanded timing of PMAD screening, which by current design typically happens in the immediate postpartum maternal inpatient hospital stay, and at outpatient obstetric and pediatric routine follow-up visits [19, 30]. Because PMAD symptoms can evolve over time, serial screening is important for NICU families. However, parents of preterm infants may miss some, or all, of the AAP recommended PPD screenings at 1-, 2-, 4-, and 6-month well child visits, as their infants may still be admitted to the NICU. Additionally, outpatient pediatric visits following NICU discharge may have their focus pulled toward the on-going medical care needs of the infant rather than PMAD screening. Up to $40 \%$ of women miss their routine postpartum obstetric appointment with its associated PMAD screening opportunity [30]. Of these women, a disproportionate number are women of color, low economic status, and those with high life stressors, such as having a baby in the NICU [30]. Repeated screening of parents during high-risk pregnancy and while their child is still inpatient would improve catchment of families' developing PMAD symptoms related to the changing stressors of parenting a child with complex medical needs.

Thirdly, we propose that screening programs for PMAD extend beyond biological mothers to include other primary caregivers. Our NICU families are varied in make-up with primary caregivers including fathers, non-gestational mothers, grandparents, planned adoptive parents, and others. We have found that no primary caregiver escapes the toll of loving a critically ill infant. Fathers, in particular, experience significant stress and mental health burden during the postpartum period. Studies specific to NICU parents have highlighted both the frequency of PMAD symptoms in NICU fathers as well as the differences between NICU fathers and mothers in terms of PMAD severity and duration [5, 8, 9, 29, 31-34]. While recommendations exist to assess fathers' mental health and adjustment to parenting $[19,35]$, in our experience this is not often being translated into action. We suggest that PMAD screening is applicable to any adult who is acting in a parental role to a hospitalized neonate.

\section{USING EXISTING RESOURCES TO IMPLEMENT PMAD SCREENING, REFERRAL, AND TREATMENT IN THE NICU}

Improved awareness of and broader screening for PMAD in the NICU need to be accompanied by a stronger system of referral and treatment to achieve the positive progress we hope to see. Discrete steps toward building this system are outlined in the "Consensus Bundle on Maternal Mental Health: Perinatal Depression and Anxiety [17]." These core steps include identifying the right mental health screening tools, educating staff on how and when to use the screening tools, establishing a protocol for responding to positive screens by employing local mental health support resources, having an emergency response system for any positive screens indicating suicidality or threat of harm to others, implementing a systematic review process to address balancing measures for quality improvement, and continuing efforts toward a local culture of knowledge and acceptance of PMAD and its significance [17]. To support a NICU team in moving through these steps, we will touch on available tools that can be employed in these efforts and highlight several recommendations from previous research.

While there is no single, comprehensive PMAD screening questionnaire, there are several available tools that can be used in concert. The Edinburgh Postnatal Depression Scale (EPDS) screens for both depression and anxiety symptoms, excludes constitutional symptoms such as changes in sleep that are universal to new parents, and has been translated into 50 languages. The Patient Health Questionnaire (PHQ9), General Anxiety Disorder (GAD7) screen, and Primary Care PTSD Screen (PC-PTSD-5) are designed for use in the general population to screen for depression, anxiety, and PTSD, respectively, but have each been validated specifically for the perinatal population, as well $[9,36-38]$. Each of these screens takes less than 5 minutes to complete and has high sensitivity and specificity for identifying its targeted condition(s) [9].

Ideally, PMAD screening would start in the antepartum period for families anticipated to have a child admitted to the NICU [14, 39-41]. With or without an antenatal meeting, NICU mental health support staff should meet with each NICU family in the first 72 hours of a child's admission to (re)establish a working relationship, normalize emotional distress, and identify additional PMAD risk factors [42]. At a minimum, formal parental PMAD screening should then take place during the first week of a child's admission, 1-2 days prior to anticipated NICU discharge for admissions greater than 1-2 weeks, and during NICU developmental follow-up visits in the first year 
after NICU discharge $[3,10,12,42,43]$. Additional screening may be necessary for families with more risk factors.

The NICU team will need to build a system for reviewing screens, and then providing those who screen positive with appropriate referrals for mental health support services. Note that previous studies have shown that the majority of women who screen positive for a postpartum mental health disorder do not utilize their mental health referral, in part because referrals to outside mental health providers are often perceived by families as added stress, requiring extra appointments at additional facilities $[4,9,11,42,44,45]$. Psychologists and psychiatrists dedicated to the treatment of NICU parents and working within the NICU itself could, therefore, greatly improve referral uptake. In fact, prior studies evaluating the benefit of psychotherapy conducted within the physical space of the NICU have shown significant reduction in PMAD symptoms in parents [33, 42].

This call for a stronger mental health support team within the $\mathrm{NICU}$ is not novel. A multidisciplinary workgroup of NICU clinical care providers and NICU parents representing 29 professional and parent groups and 22 academic institutions came together through the National Perinatal Association to discuss psychosocial support for parents of NICU infants [39, 42]. They published recommendations in a supplemental issue of the Journal of Perinatology, including that NICUs with 20 or more beds should employ at least one full-time master's level social worker, one parttime doctoral level psychologist, and should consider employing a part-time psychiatrist and psychiatric nurse [42]. This type of perinatal mental health workforce could potentially facilitate PMAD screening, counseling, and potential treatment for NICU families in addition to educational initiatives for NICU staff [42].

The funding allocated for NICU mental health professionals and rates of insurance reimbursement vary between healthcare systems and states and can be a significant limiting factor to implementing teams as described above. While these mental health support services are likely to be cost-effective when taking into account the broader, long-term impacts of PMADs, their funding would necessitate up-front investment from hospital administrators and would benefit from optimization of billing practices [42].

An additional way to bring mental health services to families in the NICU at a lower cost is through telehealth services. Although the merits and potential pitfalls of mental health support delivered remotely are beyond the scope of this perspective, telehealth and telepsychiatry, accelerated by the COVID-19 pandemic, have improved general access to mental health care [46-48]. Mobile applications and secure messaging platforms show promise for the digital delivery of mental health education, interventions and self-guided care [49-51]. These types of applications may prove helpful in the NICU parent population.

\section{BETTER FAMILY CARE YIELDS BETTER INFANT OUTCOMES}

Untreated PMAD symptoms come with staggering costs, as previously described. As neonatologists, we take particular interest in the global data demonstrating that perinatal mental health disorders are associated with a negative impact on developing infants' physical, socioemotional, cognitive and mental health that can be pervasive throughout their lives and even subsequently transmitted to future generations [52-59]. While all children and families could benefit from strong systems of PMAD symptom recognition and treatment, this benefit is particularly significant for children who require NICU care due to extremely premature birth or complex medical needs. These children are already at risk for poor neurodevelopment and mental health outcomes over their lifetimes, which would be compounded by untreated parental PMAD symptoms.

Perinatal mental health interventions could also be particularly impactful for NICU parents facing structural adversities, in part because PMAD disproportionately affects parents with lower socioeconomic status, poor social support, multiple gestations, and preterm infants admitted to the NICU [19, 30, 58, 60]. Recent research has evaluated disparities between preterm infants by race and ethnicity, and associated these disparities with impacts on quality of care and long-term health outcomes, with black infants most negatively affected $[60,61]$. Since the potential for a positive impact of improved PMAD identification and treatment is magnified within these groups, they should be prioritized in research initiatives.

\section{CONCLUSIONS}

We believe that increased awareness, screening, and treatment of PMADs in NICU parents have the potential to improve family-infant outcomes. To maximize this impact, we need institutional commitment from healthcare organizations, buy-in for resource mobilization at the local, state and national levels, and engagement from multidisciplinary teams invested in the hard work of structural change, particularly focused on those most underserved by the current system. We call on neonatal providers to advocate for policy changes, quality improvement initiatives, updated educational curricula for neonatal fellows, and outcomes-based research supporting PMAD treatment in NICU parents. We believe these efforts align with the mission of the AAP: to strive for optimal physical, mental, and social health from infancy into young adulthood [62].

\section{REFERENCES}

1. Paulsen M. Root cause analysis. JAMA. 2021;325:225-6.

2. Kleine I. Interventions to prevent perinatal depression: US Preventive Services Task Force Recommendation Statement. Arch Dis Child Educ Pr Ed. 2020;105:242-3.

3. Feeley N, Zelkowitz P, Cormier C, Charbonneau L, Lacroix A, Papageorgiou A. Posttraumatic stress among mothers of very low birthweight infants at 6 months after discharge from the neonatal intensive care unit. Appl Nurs Res. 2011;24:114-7.

4. Roque ATF, Lasiuk GC, Radunz V, Hegadoren K. Scoping review of the mental health of parents of infants in the NICU. J Obstet Gynecol Neonatal Nurs. 2017;46:576-87.

5. Schecter R, Pham T, Hua A, Spinazzola R, Sonnenklar J, Li D, et al. Prevalence and longevity of PTSD symptoms among parents of nicu infants analyzed across gestational age categories. Clin Pediatr. 2020;59:163-9.

6. Shaw RJ, St, John N, Lilo E, Jo B, Benitz W, Stevenson DK, et al. Prevention of traumatic stress in mothers of preterms: 6-month outcomes. Pediatrics. 2014;134: e481-8.

7. Holditch-Davis D, Miles MS, Weaver MA, Black B, Beeber L, Thoyre S, et al. Patterns of distress in African-American mothers of preterm infants. J Dev Behav Pediatr. 2009;30:193-205.

8. Lefkowitz DS, Baxt C, Evans JR. Prevalence and correlates of posttraumatic stress and postpartum depression in parents of infants in the Neonatal Intensive Care Unit (NICU). J Clin Psychol Med Settings. 2010;17:230-7.

9. Hynan MT, Mounts KO, Vanderbilt DL. Screening parents of high-risk infants for emotional distress: rationale and recommendations. J Perinatol. 2013;33:748-53.

10. Kim WJ, Lee E, Kim KR, Namkoong K, Park ES, Rha DW. Progress of PTSD symptoms following birth: a prospective study in mothers of high-risk infants. J Perinatol. 2015;35:575-9.

11. Chertok IR, McCrone S, Parker D, Leslie N. Review of interventions to reduce stress among mothers of infants in the NICU. Adv Neonatal Care. 2014;14:30-7.

12. Purdy IB, Craig JW, Zeanah P. NICU discharge planning and beyond: recommendations for parent psychosocial support. J Perinatol. 2015:35:S24-8. Suppl 1

13. Cox EQ, Sowa NA, Meltzer-Brody SE, Gaynes BN. The perinatal depression treatment cascade: baby steps toward improving outcomes. J Clin Psychiatry. 2016;77:1189-200.

14. McKee K, Admon LK, Winkelman TNA, Muzik M, Hall S, Dalton VK, et al. Perinatal mood and anxiety disorders, serious mental illness, and delivery-related health outcomes, United States, 2006-2015. BMC Women's Health. 2020;20:150.

15. Admon LK, Winkelman TNA, Zivin K, Terplan M, Mhyre JM, Dalton VK. Racial and ethnic disparities in the incidence of severe maternal morbidity in the United States, 2012-2015. Obstet Gynecol. 2018;132:1158-66.

16. Luca DL, Margiotta C, Staatz C, Garlow E, Christensen A, Zivin K. Financial toll of untreated perinatal mood and anxiety disorders among 2017 births in the United States. Am J Public Health. 2020;110:888-96.

17. Kendig S, Keats JP, Hoffman MC, Kay LB, Miller ES, Moore Simas TA, et al. Consensus bundle on maternal mental health: perinatal depression and anxiety. Obstet Gynecol. 2017;129:422-30. 
18. Bauer A, Knapp M, Parsonage M. Lifetime costs of perinatal anxiety and depression. J Affect Disord. 2016;192:83-90.

19. Earls MF, Yogman MW, Mattson G, Rafferty J, Committee on Psychosocial Aspects of $\mathrm{C}$, Family $\mathrm{H}$. Incorporating recognition and management of perinatal depression into pediatric practice. Pediatrics. 2019;143:e20183259.

20. Clement S, Schauman O, Graham T, Maggioni F, Evans-Lacko S, Bezborodovs N, et al. What is the impact of mental health-related stigma on help-seeking? A systematic review of quantitative and qualitative studies. Psychol Med. 2015;45:11-27.

21. Wei Y, McGrath PJ, Hayden J, Kutcher S. Mental health literacy measures evaluating knowledge, attitudes and help-seeking: a scoping review. BMC Psychiatry. 2015;15:291.

22. Hall SL, Famuyide ME, Saxton SN, Moore TA, Mosher S, Sorrells K, et al. Improving staff knowledge and attitudes toward providing psychosocial support to NICU parents through an online education course. Adv Neonatal Care. 2019;19:490-9.

23. Scala M, Marchman, V, Dowtin, L, Givrad, S, Nguyen, T, Thomson, A, et. al. Evaluation of a course for neonatal fellows on providing psychosocial support to NICU families. Poster presented at: Pediatric Academic Societies Conference; 2021 April 30-May 4; virtual format.

24. Sanders MR, Hall SL. Trauma-informed care in the newborn intensive care unit: promoting safety, security and connectedness. J Perinatol. 2018;38:3-10.

25. Coughlin M. Trauma-informed care in the NICU. New York, NY: Springer Publishing Company, LLC; 20172017.

26. Pediatrics ABo. Foundation Strategic Plan. 2021. https://www.abp.org/foundation/ roadmap.

27. Education ACOGM. ACGME program requirements for graduate medical education in neonatal-perinatal medicine ACGME.ORG: ACGME; 2020. https://www.acgme.org/ Portals/0/PFAssets/ProgramRequirements/329_NeonatalPerinatalMedicine_2020. pdf?ver=2020-06-29-162707-410.

28. Yeaton-Massey A, Herrero T. Recognizing maternal mental health disorders: beyond postpartum depression. Curr Opin Obstet Gynecol. 2019;31:116-9.

29. Shaw RJ, Bernard RS, Deblois T, Ikuta LM, Ginzburg K, Koopman C. The relationship between acute stress disorder and posttraumatic stress disorder in the neonatal intensive care unit. Psychosomatics. 2009;50:131-7.

30. Committee on Obstetric P. The American College of Obstetricians and Gynecologists Committee Opinion no. 630. Screening for perinatal depression. Obstet Gynecol. 2015;125:1268-71.

31. Garfield CF, Lee YS, Warner-Shifflett L, Christie R, Jackson KL, Miller E. Maternal and paternal depression symptoms during NICU stay and transition home. Pediatrics. 2021;148:e2020042747.

32. Pace CC, Spittle AJ, Molesworth CM, Lee KJ, Northam EA, Cheong JL, et al. Evolution of depression and anxiety symptoms in parents of very preterm infants during the newborn period. JAMA Pediatr. 2016;170:863-70.

33. Sabnis A, Fojo S, Nayak SS, Lopez E, Tarn DM, Zeltzer L. Reducing parental trauma and stress in neonatal intensive care: systematic review and meta-analysis of hospital interventions. J Perinatol. 2019;39:375-86.

34. Turan T, Basbakkal Z, Ozbek S. Effect of nursing interventions on stressors of parents of premature infants in neonatal intensive care unit. J Clin Nurs. 2008; 17:2856-66.

35. Walsh TB, Davis RN, Garfield C. A call to action: screening fathers for perinatal depression. Pediatrics. 2020;145:e20191193.

36. Prins A, Bovin MJ, Smolenski DJ, Marx BP, Kimerling R, Jenkins-Guarnieri MA, et al. The primary care PTSD screen for DSM-5 (PC-PTSD-5): development and evaluation within a veteran primary care sample. J Gen Intern Med. 2016;31:1206-11.

37. Spitzer RL, Kroenke K, Williams JB, Lowe B. A brief measure for assessing generalized anxiety disorder: the GAD-7. Arch Intern Med. 2006;166:1092-7.

38. Wenz-Gross M, Weinreb L, Upshur C. Screening for post-traumatic stress disorder in prenatal care: prevalence and characteristics in a low-income population. Matern Child Health J. 2016;20:1995-2002.

39. Hynan MT, Hall SL. Psychosocial program standards for NICU parents. J Perinatol. 2015;35:S1-4. Suppl 1

40. Puryear L, Nong YH, Correa NP, Cox K, Greeley CS. Outcomes of implementing routine screening and referrals for perinatal mood disorders in an integrated multi-site pediatric and obstetric setting. Matern Child Health J. 2019;23:1292-8.

41. Accortt EE, Wong MS. It is time for routine screening for perinatal mood and anxiety disorders in obstetrics and gynecology settings. Obstet Gynecol Surv. 2017;72:553-68.

42. Hynan MT, Steinberg Z, Baker L, Cicco R, Geller PA, Lassen S, et al. Recommendations for mental health professionals in the NICU. J Perinatol. 2015;35:S14-8. Suppl 1

43. Penny KA, Friedman SH, Halstead GM. Psychiatric support for mothers in the Neonatal Intensive Care Unit. J Perinatol. 2015;35:451-7.

44. Xue WQ, Cheng KK, Xu D, Jin X, Gong WJ. Uptake of referrals for women with positive perinatal depression screening results and the effectiveness of interventions to increase uptake: a systematic review and meta-analysis. Epidemiol Psychiatr Sci. 2020;29:e143.
45. Hall SL, Cross J, Selix NW, Patterson C, Segre L, Chuffo-Siewert R, et al. Recommendations for enhancing psychosocial support of NICU parents through staff education and support. J Perinatol. 2015;35:S29-36. Suppl 1

46. Chakrabarti S. Usefulness of telepsychiatry: a critical evaluation of videoconferencingbased approaches. World J Psychiatry. 2015;5:286-304.

47. Cowan KE, McKean AJ, Gentry MT, Hilty DM. Barriers to use of telepsychiatry: clinicians as gatekeepers. Mayo Clin Proc. 2019;94:2510-23.

48. Hubley S, Lynch SB, Schneck C, Thomas M, Shore J. Review of key telepsychiatry outcomes. World J Psychiatry. 2016;6:269-82.

49. Patel S, Saunders KE. Apps and wearables in the monitoring of mental health disorders. Br J Hosp Med. 2018;79:672-5.

50. Bucci S, Schwannauer M, Berry N. The digital revolution and its impact on mental health care. Psychol Psychother. 2019;92:277-97.

51. Hussain-Shamsy N, Shah A, Vigod SN, Zaheer J, Seto E. Mobile health for perinatal depression and anxiety: scoping review. J Med Internet Res. 2020;22:e17011.

52. Fernandez y Garcia E, Joseph J, Wilson MD, Hinton L, Simon G, Ludman E, et al. Pediatric-based intervention to motivate mothers to seek follow-up for depression screens: The Motivating Our Mothers (MOM) trial. Acad Pediatr. 2015;15:311-8.

53. Hoffman C, Dunn DM, Njoroge WFM. Impact of postpartum mental illness upon infant development. Curr Psychiatry Rep. 2017;19:100.

54. Ramchandani PG, Psychogiou L, Vlachos H, lles J, Sethna V, Netsi E, et al. Paternal depression: an examination of its links with father, child and family functioning in the postnatal period. Depress Anxiety. 2011;28:471-7.

55. McManus BM, Poehlmann J. Maternal depression and perceived social support as predictors of cognitive function trajectories during the first 3 years of life for preterm infants in Wisconsin. Child Care Health Dev. 2012:38:425-34.

56. Keim SA, Daniels JL, Dole N, Herring AH, Siega-Riz AM, Scheidt PC. A prospective study of maternal anxiety, perceived stress, and depressive symptoms in relation to infant cognitive development. Early Hum Dev. 2011;87:373-80.

57. Barlow J, Bennett C, Midgley N, Larkin SK, Wei Y. Parent-infant psychotherapy for improving parental and infant mental health. Cochrane Database Syst Rev. 2015;1:CD010534.

58. Stein A, Pearson RM, Goodman SH, Rapa E, Rahman A, McCallum M, et al. Effects of perinatal mental disorders on the fetus and child. Lancet 2014;384:1800-19.

59. O'Donnell KJ, Meaney MJ. Fetal origins of mental health: the developmental origins of health and disease hypothesis. Am J Psychiatry. 2017;174:319-28.

60. Sidebottom A, Vacquier M, LaRusso $E$, Erickson D, Hardeman R. Perinatal depression screening practices in a large health system: identifying current state and assessing opportunities to provide more equitable care. Arch Womens Ment Health. 2021;24:133-44.

61. Sigurdson K, Mitchell B, Liu J, Morton C, Gould JB, Lee HC, et al. Racial/ethnic disparities in neonatal intensive care: a systematic review. Pediatrics. 2019;144: e20183114.

62. Hagan JF, Shaw JS, Duncan PM, editors. Bright Futures: Guidelines for Health Supervision of Infants, Children, and Adolescents. 4th ed. Elk Grove Village, IL: American Academy of Pediatrics; 2017 Page v.

\section{ACKNOWLEDGEMENTS}

MP has been supported by the National Institute of Health Building Interdisciplinary Research Careers in Women's Health (BIRCWH) grant 5K12HD055887-14(MPI); however, the NIH had no role in the design and conduct of this perspective piece.

\section{AUTHOR CONTRIBUTIONS}

All authors contributed equally to this work, approved the final manuscript as submitted, and agreed to be accountable for all aspects of the work.

\section{COMPETING INTERESTS}

The authors declare no competing interests.

\section{ADDITIONAL INFORMATION}

Correspondence and requests for materials should be addressed to Julie Johnson Rolfes.

Reprints and permission information is available at http://www.nature.com/ reprints

Publisher's note Springer Nature remains neutral with regard to jurisdictional claims in published maps and institutional affiliations. 